\title{
Strength of Dissimilar Materials Joint between A5052 and PET Joined by Using Friction Stir Heating*
}

\author{
Farazira YUSOF**, Yukio MIYASHITA**, Ryosuke OZAKI**, \\ Nobushiro SEO***, Yuichi OTSUKA** and Yoshiharu MUTOH** \\ **Nagaoka University of Technology \\ Nagaoka, Niigata, 940-2188, Japan \\ E-mail: miyayuki@mech.nagaokaut.ac.jp \\ *** Nippon Light Metal Co. Ltd. \\ Shizuoka, Shizuoka, Japan
}

\begin{abstract}
A new direct joining method for dissimilar materials between a metal and a plastic by using friction stir heating technique is introduced. A5052 and polyethylene terephthalate (PET) were used as materials used in this study. The joining strength was evaluated under tensile shear loading condition. The fracture surface and the cross section of the joined specimens were observed in order to understand characteristics of the joints. The results show that A5052 and PET were successfully welded by the present joining method. Similar strength of the joints was obtained regardless of the joining conditions. Melting and softening behaviors of PET and formation of bubbles observed in welded area might affect the joining strength.
\end{abstract}

Key words: Dissimilar Materials Joint, PET, Aluminum Alloy, Friction Stir Heating

\section{Introduction}

The application of light metals such as aluminum or magnesium alloy in automotive parts is very effective for the weight reduction purposes. In addition, efficient fuel consumption can be benefit from the reduction of the weight. Besides that, plastic has been used in the automotive parts due to good physical properties such as excellent corrosion resistance, durability, insulation and lightweight. The both dissimilar materials are suitable to be coupled in the production of automotive parts. Unfortunately the combination of metals and plastics in the products assembly makes them difficult to join together due to unlike material properties. Mechanical fastening or adhesive has been mainly applied to join between a metal and a plastic. However these techniques have limitations, for example it is difficult to join a product with intricate shape, longer assembly time, and required much consumable. Moreover, the use of screws or rivets may increase weight of engineering products. Hence an appropriate joining technique should be proposed to improve quality, assembly time and reliability of the dissimilar materials joint. Some research works on dissimilar materials direct joining between a metal and a plastic have been reported ${ }^{(1)-(4)}$. In basically, necessary joining process is summarized as two processes, (1) softening or melting plastic and (2) pushing them on to the metal surface. In case of heat energy applies separately by using a heat source, another apparatus or jig is needed to apply the mechanical pushing force to the interface. On the other hand, it could be interesting and effective if

*Received 19 July, 2011 (No. 11-0421) [DOI: 10.1299/jmmp.5.1051]

Copyright $\odot 2011$ by JSME 
those two processes are realized in the same time during the joining process. Friction stir welding (FSW) is a solid state joining process. The joining process does not involve any use of filler material, shielding gas or solvents ${ }^{(5)}$. Moreover, this joining technique can be applied to various types of joint like butt joints, lap joints, $\mathrm{T}$ butt joints and fillet joint ${ }^{(6)}$. During FSW process in lap joint, heat energy is generated and pressure is applied to the interface in the same time.

In the present study, dissimilar materials joining between aluminum alloy (A5052) and polyethylene terephthalate (PET) using friction stir heating technique was studied. Effects of joining parameters, such as plunge depth and plunge speed on the strength and joining mechanism are discussed.

\section{Experimental Procedure}

The materials used were A5052 aluminum alloy and polyethylene terephthalate (PET) sheets with thickness of $3 \mathrm{~mm}$. A5052 as rolled plate was used and its surface roughness was $R_{a}=0.31 \mu \mathrm{m}$. Surfaces of the materials used were cleaned by using acetone for A5052 and ethanol for PET before the joining test. Physical and mechanical properties of A5052 and PET are shown in Table 1. Rectangular specimens with size of $40 \mathrm{~mm} \times 100 \mathrm{~mm}$ were prepared for the joining experiment. The welding process in the present study is schematically shown in Fig. 1. In this study, the lap joint was produced. The A5052 sheet was placed on the top of the PET sheet. The rotating tool consists of a shoulder with $\phi 8 \mathrm{~mm}$ in diameter and a small conical center probe. In the process, a rotating tool partially penetrates

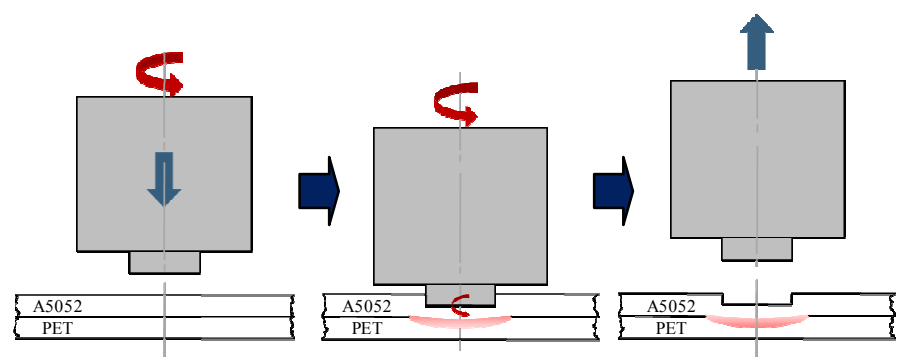

Fig.1 Schematic illustration of the welding process between A5052 and PET.

Table 1 Physical and mechanical properties for A5052 and PET.

\begin{tabular}{lcc}
\hline & $\mathbf{A 5 0 5 2}$ & PET \\
\hline Density, g/cm & & \\
Glass transition temperature, ${ }^{\circ} \mathrm{C}$ & 2.68 & 1.45 \\
Melting temperature, ${ }^{\circ} \mathrm{C}$ & - & 80 \\
Specific heat capacity, $\mathrm{J} / \mathrm{g}^{\circ} \mathrm{C}$ & $607-649$ & $200-255$ \\
Thermal conductivity, $\mathrm{W} / \mathrm{m}^{\circ} \mathrm{C}$ & 0.88 & 1.00 \\
Tensile strength, $\mathrm{MPa}$ & 138 & 0.24 \\
Proof stress & 260 & 49 \\
Elastic modulus, $\mathrm{GPa}$ & 215 & - \\
\hline
\end{tabular}

Table 2Welding parameters applied in the present study.

\begin{tabular}{lc}
\hline Parameters & Value applied \\
\hline Rotating speed , r.p.m. & 3,000 \\
Plunge depth $\left(D_{p}\right), \mathrm{mm}$ & 0.4 and 0.7 \\
Plunge speed , mm/min & $5,10,20$, and 40 \\
Holding time at the plunge depth, $\mathrm{s}$ & 2 \\
\hline
\end{tabular}


from the top surface of A5052 until a certain plunge depth. Then a tool kept rotating at the depth for the holding time. Finally, a tool was taken out from an aluminum alloy sheet. In the joining experiment, rotating speed, plunge speed, plunge depth and holding time were controlled as presented in Table 2. In order to evaluate the joining strength, tensile shear test was carried out with loading rate of $0.5 \mathrm{~mm} / \mathrm{min}$ under displacement control. The geometry and dimensions of the shear tensile specimen is illustrated in Fig. 2. Supporting plates with thickness of $3 \mathrm{~mm}$ were attached at both gripping parts of the joint to adjust offset of the loading center line in the tensile shear test. Distance between clamping jigs was $100 \mathrm{~mm}$.

\section{Result and discussion}

An example of observation of welded region is shown in Fig. 3. A circle in the figure shows area of molten or softened PET and is defined as welded area in the present study. At the center of welded area, formation of bubbles is observed. An example of cross-sectional views of the joint is shown in Fig. 4. According to the figure, A5052 deformed and bubbles were formed near the region pushed by the tool in PET side. In this region, molten or softened PET was tightly attached to A5052 as shown in enlarged pictures of the cross section in bottom of Fig.4. It is believed that PET might melt during the joining process and heat generated at the interface might be high enough to induce bubbles formation in the PET. Welded area was measured by observation of the interface from PET side since its transparency, where the welded area is defined as an area of molten or softened PET (shown as a circle in Fig.3). Effect of plunge speed on the welded area is shown in Fig. 5. In case of lower plunge speed conditions ( 5 and $10 \mathrm{~mm} / \mathrm{min}$ ), the result shows that the welded area decreased with increasing plunge speed for both cases of plunge depth, $D_{p}=0.4$ and $0.7 \mathrm{~mm}$. In comparison of the welded area between plunge depth $D_{p}=0.4$ and $0.7 \mathrm{~mm}$, the welded area obtained with deeper plunge depth was larger than that obtained with shallower plunge

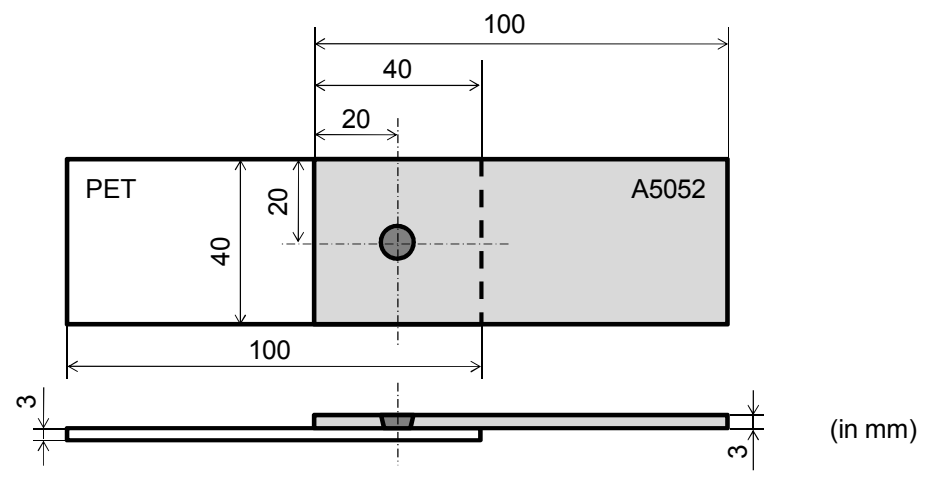

Fig.2 Joint specimen for tensile shear test.

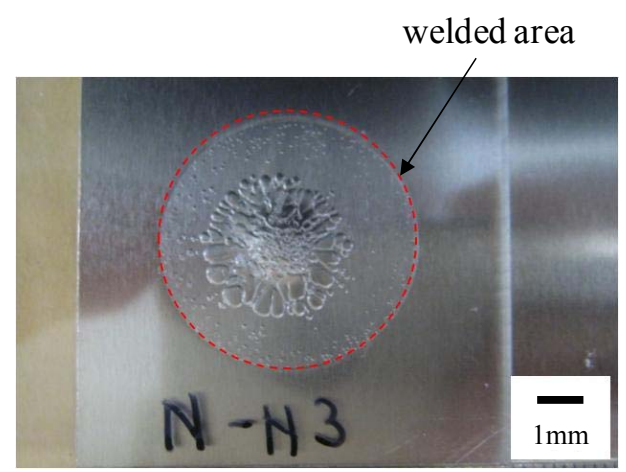

Fig.3 Observation of the welded area from PET side. (plunge depth; $0.4 \mathrm{~mm}$, plunge speed; $10 \mathrm{~mm} / \mathrm{min}$ ) 
depth. In the present study, heat generated during the joining process was spent to softening and melting of PET, then they were pushed on to the metal surface. There are three possible heat sources produced in the process that are friction work between a tool and top surface of the specimen, friction work at the interface of top and bottom specimen, and plastic deformation of the material ${ }^{(7)}$. In case of deeper plunge depth condition, large heat energy can be generated during the plunging process, because the total time for the plunging process is longer. Then larger welded area was obtained with deeper plunge depth condition. On the other hand, since heat generation for a unit plunge depth is considered as the same under the same plunge speed condition, decreasing rate of the welded area due to increasing plunge speed (shown as a broken line in Fig.5) is almost the same regardless of the difference in total plunge depth, $D_{p}$. It is also speculated that slower plunge speed induced more heat generation at the joined interface due to ample time for the tool to rotating in the material thus prolonged heat generation. Mokhtar Awang and Victor H. Mucino have elaborated the effect of plunge speed on energy generation during FSSW (Friction Stir Spot Welding) process. They reported that the frictional dissipation energy in $5 \mathrm{~mm} / \mathrm{s}$ plunge speed reduces $29 \%$ from the frictional dissipation heat in $1 \mathrm{~mm} / \mathrm{s}$ plunge speed. Similarly when they increased the plunge speed into $10 \mathrm{~mm} / \mathrm{s}$ the heat generated was decreased by $14 \%{ }^{(7)}$. In the

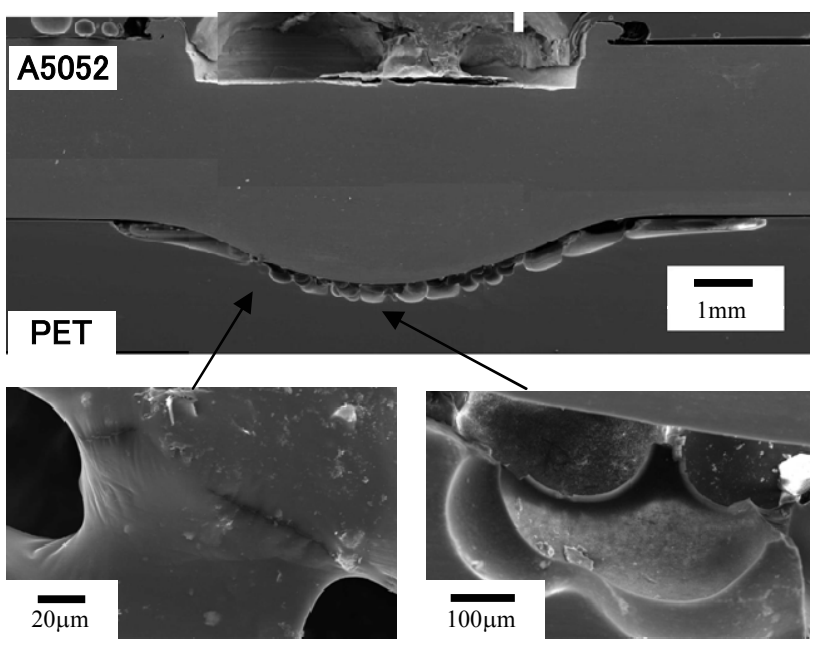

Fig.4 Cross sectional observation at the interface of the joint. (plunge depth; $0.7 \mathrm{~mm}$, plunge speed; $20 \mathrm{~mm} / \mathrm{min}$ )

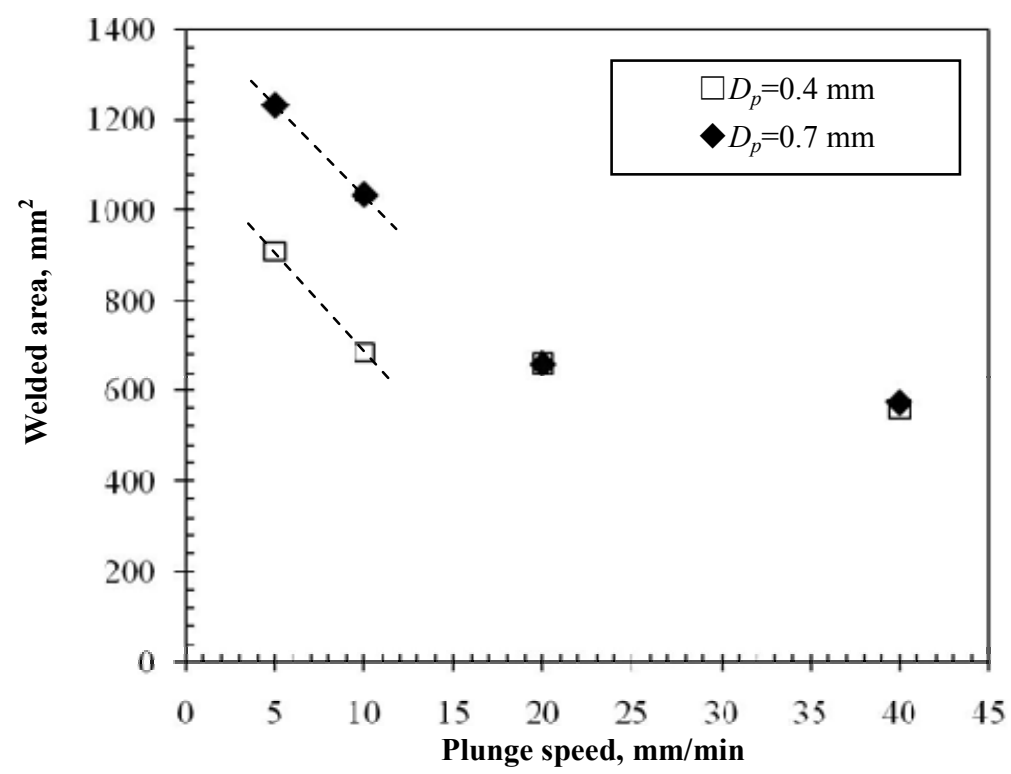

Fig.5 Effect of plunge speed on the welded area. 
present result shown in Fig.5, in case of higher plunge speed conditions (20 and 40 $\mathrm{mm} / \mathrm{min}$ ), it was found that the welded area for specimens welded at plunge speed of 20 $\mathrm{mm} / \mathrm{min}$ and $40 \mathrm{~mm} / \mathrm{min}$ were almost the same even in different plunge depths $\left(D_{p}=0.4\right.$ and $0.7 \mathrm{~mm}$ ). The generated heat during the plunging process was small in condition of higher plunge speed, because less time for the plunging. The heat energy could not be enough to heat for large area of the interface. Then only PET in relatively small area under the tool could be possible to softening and melting, and the area was pushed on to the metal surface to form the welded area.

Effect of plunge speed on tensile shear failure load of the joint is shown in Fig. 6. All joints were broken at the maximum tensile shear load. According to Fig.6, tensile shear failure load decreased with increasing plunge speed in specimen welded at plunge depth, $D_{p}$ $=0.7 \mathrm{~mm}$ within the lower plunge speed conditions ( 5 and $10 \mathrm{~mm} / \mathrm{min}$ ). It is speculated that lower plunge speed provides sufficient heat to be formed at the interface thus enlarge welded area and simultaneously increased failure load. In contrast, the joined specimens

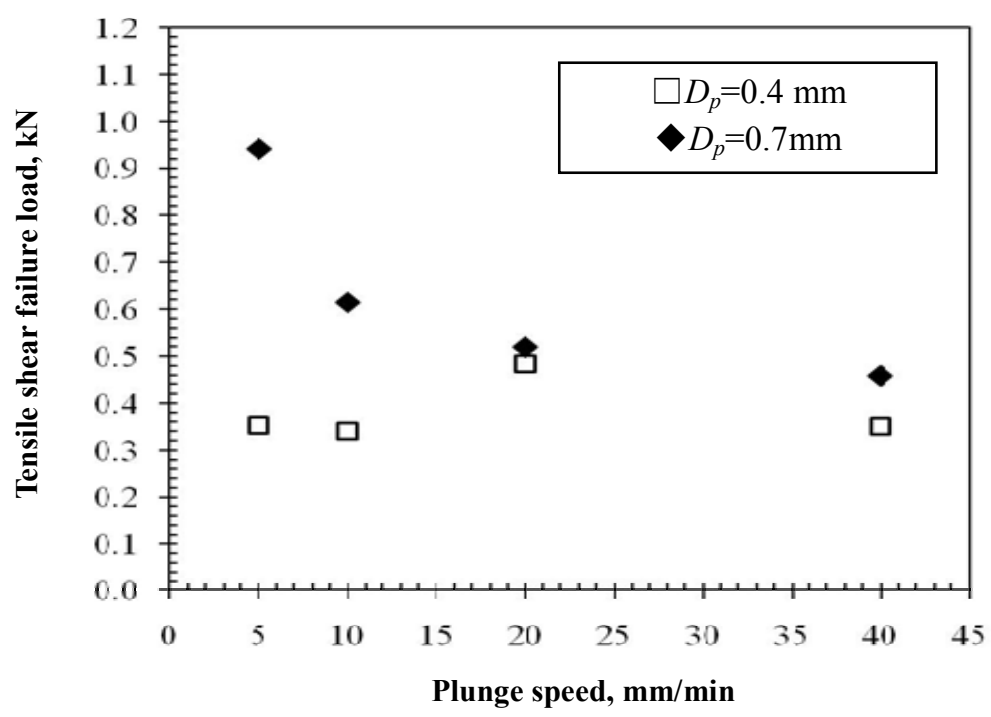

Fig.6 Effect of plunge speed on tensile shear failure load.

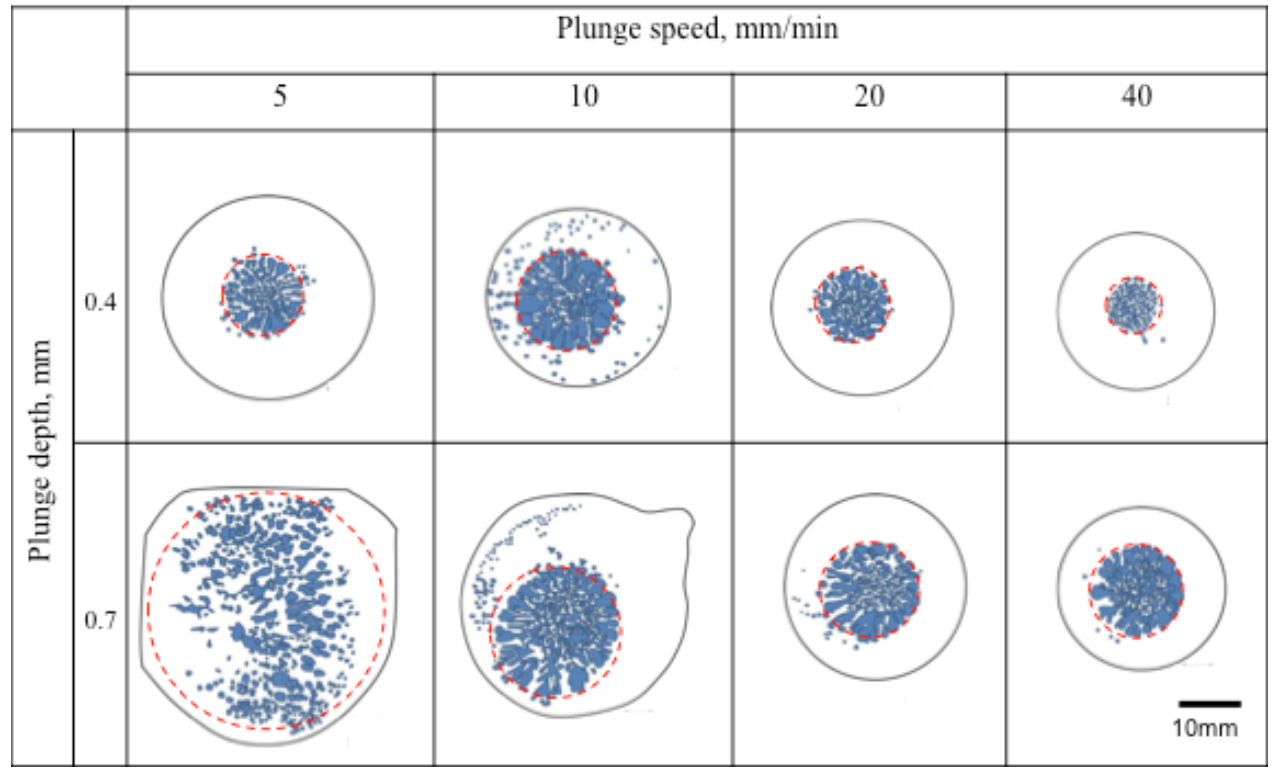

Fig.7 Sketches of bubble formation at the interface. 
welded at plunge depth, $D_{p}=0.4 \mathrm{~mm}$ have resulted almost constant failure load regardless of plunge speed. It is believed that formation of bubbles in the welding region may influence the tensile shear failure load of the joined specimens. Therefore, the illustrations of the bubbles distribution in the joined specimens observed from PET side are shown in Fig. 7. The outer solid line in the figure shows area of molten or softened PET. It was observed that the most of the bubbles were mainly formed in the center (tool area) of the welded area and this indicates high heat was generated during tool rotating. In case of the specimen welded at low plunge depth $\left(D_{p}=0.4 \mathrm{~mm}\right)$, the bubbles were rather small and the bubbles were mainly accumulated at the tool region for all specimens. Meanwhile for the specimen welded at deeper plunge depth $\left(D_{p}=0.7 \mathrm{~mm}\right)$, the size of bubbles were randomly mixed and the bubbles were densely distributed in the welding region especially for joined specimens welded at plunge speed of 5 and $10 \mathrm{~mm} / \mathrm{min}$. It is considered that bubble

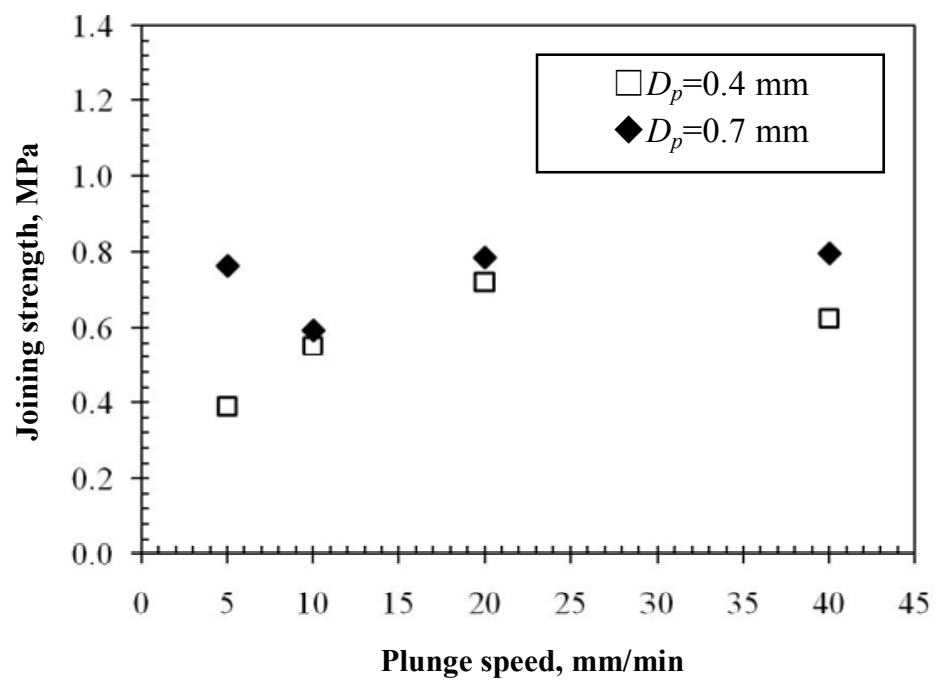

Fig.8 Effect of plunge speed on tensile shear strength.

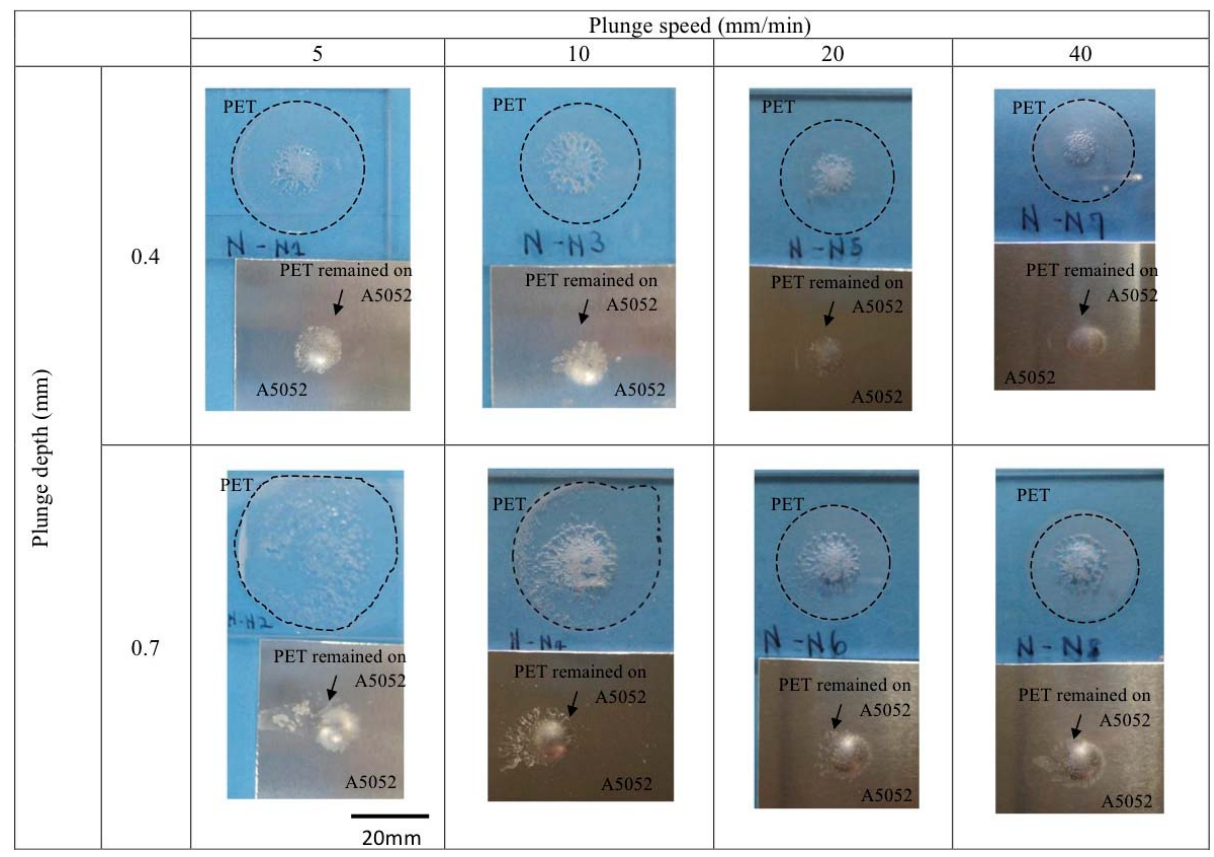

Fig.9 Observations of fracture surfaces after tensile shear test. Dashed line in the figure shows the welded area. 
nucleation during the welding process may influence the shear failure load of the joined specimens. Katayama et. al show mechanism of laser welding between a plastic and a metal $^{(2)}$. Bubbles formed during laser welding process induce high pressure and push softened and molten plastics on to metal surface. The effect of variations of plunge speed on the joining strength is shown in Fig. 8. The strength shown in Fig. 8 was calculated by the failure load divided by size of the welded area. In the figure, strengths of the joints were very low compared to tensile strength of the PET (49 MPa in the catalog data), even if the difference of loading mode is taken into account. It will need to obtain large welding area to carry the load applied in a practical application. On the other hand, this joining method is still applicable to functional application, such as protection from chemical attacking, insulation of electricity, sealing purpose, etc. Figure 8 shows that joints possessed the similar joining strength regardless of the plunge speed and the plunge depth. However, it is speculated that the joining strength in the welded area is not uniform, according to fracture surface observations as shown in Fig. 9. It was observed from the figure, PET was tightly attached around the tool region on the A5052 surface. It is presumed that an intimate contact occurred at these regions and contributed effectively on the joining strength of the joint. This is a possible reason to obtain the similar joining strength for the joints welded with plunge depth of $D_{p}=0.4 \mathrm{~mm}$, even size of the apparent welded area were varied but in small variation of the failure load.

Strength of the joint was calculated again by that dividing the failure load by the size of bubbles formed area as shown inner dashed line in Fig.7. Relationship between the strength calculated by the area of bubbles formed and the plunge speed is shown in Fig.10. The strengths calculated by using the area of bubbles formed are still lower compared to strength of the base materials. According to Fig.10, the strength increased with increase in the plunge speed for both plunge depth $D_{p}=0.4$ and $0.7 \mathrm{~mm}$. Higher strength was obtained for joints joined at $D_{p}=0.4 \mathrm{~mm}$ compared to the joints joined at $D_{p}=0.7 \mathrm{~mm}$. These results mean that the strength decreased with increase in heat input. Higher temperature at the joining area might be induced by higher heat input and could lead formation of bubbles significantly. Difference in joining strength at different place inside of the welded area is not clear, however, it is considered that exact welded area might be not defined only by the area of bubbles formed and the role of bubbles could not be only increasing the strength.

Higher heat input, namely, lower plunge speed, may induce significant bubbles formation. The formation of bubbles can realize good contact at the interface and results in

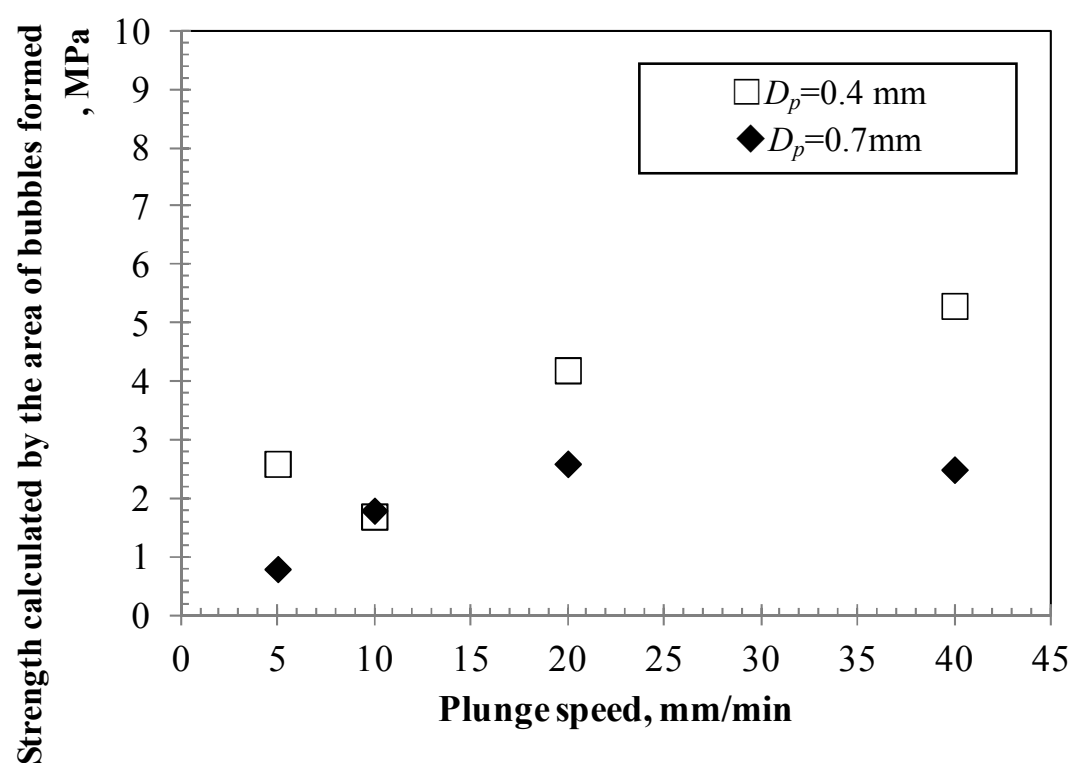

Fig.10 Joining strength calculated by the area of bubbles formed. 
increasing joining strength. However in the same time, they also play as defects and decrease the strength. Heat input should be controlled to obtain an appropriate condition. In order to improve the joining strength by using an additional factor, surface modification will be a promising way, according to the joining mechanism discussed in the present study. Effect of surface condition on the joining strength will be shown in the future work. In another point of view for practical application, varying of strength was also important. In the present work, number of the tests was limited. It could be also necessary to evaluate stability and variation of the strength in the joint produced by the present method.

\section{Summary}

This study has determined the use of friction stir heating technique in the joining between A5052 and PET. In this study, the dissimilar material joint between A5052 and PET could successfully be produced using the present welding process. The joining strength obtained in this study was almost constant regardless of welding conditions and it was fall between $0.5 \mathrm{MPa}-0.8 \mathrm{MPa}$. It was also found that the bubbles formation on vicinity of tool. It is believed that size and distribution of the bubbles would affect the joining strength.

\section{References}

(1) Sebastian KRUGER, Guntram WAGNER and Dietmar EIFLER, Ultrasonic Welding of Metal / Composite Joint, Advanced Engineering Materials, Vol.6, No.3 (2004), pp.157-159.

(2) Seiji Katayama Yousuke Kawahito, Yusuke Niwa, Akiko Tange and Shyuji Kubota, Laser Direct Joining between Stainless Steel and Amorhous Polyamide Plastic - Laser Assisted Metal - Plastic (LAMP) Joining -, Quarterly Journal of Japan Welding Society, Vol.25, No.2 (2007), pp.316-322 (in Japanese).

(3) Yukio Miyashita, Masaru Takahashi, Masashi Takemi, Kosei Oyama, Yoshiharu Mutoh and Hironori TANAKA, Dissimilar Materials Micro Welding between Stainless Steel and Plastics by Using Pulse YAG Laser, Journal of Solid Mechanics and Materials Engineering, vol.3, No.2, (2009), pp.409-415.

(4) Farazila Yusof, Yoshiharu Mutoh, Yukio Miyashita, Effect of pre-oxidized CuO layer in joining between polyethyleneterephalate (PET) and copper $(\mathrm{Cu})$ by using pulsed Nd:YAG laser, Advanced Materials Research vols.129-131, (2010), pp. 714-718.

(5) R.S. Mishra, Z.Y. Ma, Friction stir welding and processing, Materials Science and Engineering R50, (2005), pp. 1- 78.

(6) C.J. Dawes, W.M. Thomas, Friction stir process welds aluminium alloys, Welding Journal, Vol 75, 33, (1996), pp. 41- 45.

(7) Mokhtar Awang, Victor H. Mucino, Energy generation during friction stir spot welding (FSSW) of Al 6061-T6 plates, Materials and Manufacturing Processes, 25:1, (2010), pp. 167- 174 . 